\title{
Österreichischer Dermatologen-Preis 2016 verliehen
}

Zwei junge Forscher der MedUni Wien erhielten dieses Jahr den von Unilever gestifteten und am 25. November im Hotel Sacher im Rahmen der Jahrestagung der Österreichischen Gesellschaft für Dermatologie und Venerologie (ÖGDV) verliehenen Österreichischen Dermatologen-Preis. Der Mediziner Ass.-Prof. Dr. Patrick M. Brunner erhielt Auszeichnung und Preisgeld für seine herausragenden Arbeiten zur Therapie des atopischen Ekzems. Die Biologin und Genetikerin Beate M. Lichtenberger, PhD, forschte auf dem Gebiet der Ausdifferenzierung unterschiedlicher Fibroblastenzellen.

\section{Bedeutende Forschungsarbeiten} Schon zum 44. Mal werden mit dem Österreichischen Dermatologen-Preis die bedeutendsten Forschungsarbeiten auf dem Gebiet der Haut- und Venenerkrankungen gewürdigt. Die mit 5200 Euro dotierte Auszeichnung für wissenschaftliche Arbeiten wird seit

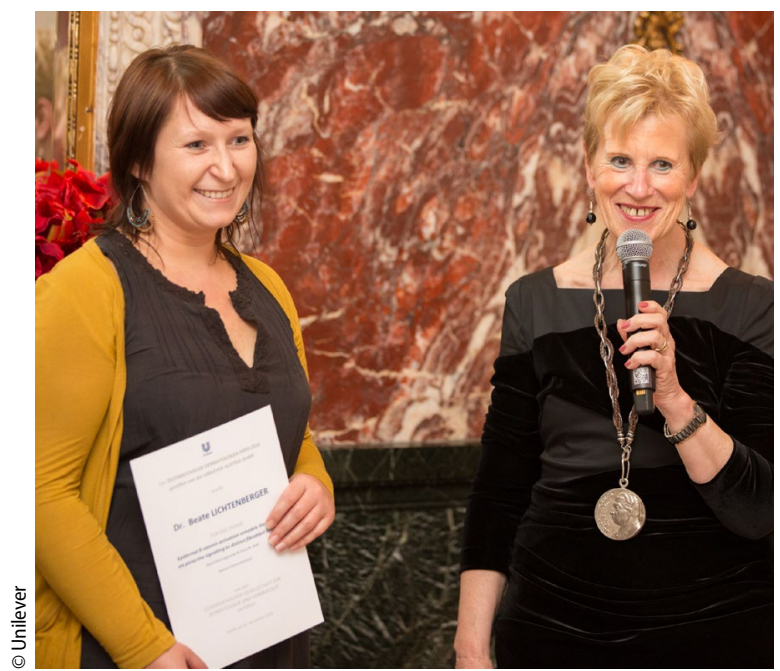

A Ph.D. Beate M. Lichtenberger und Prof. Dr. Angelika Stary, Präsidentin des ÖGDV
1973 von Unilever Austria gestiftet. "Grundlagenforschung und innovative experimentelle Fragestellungen stehen im Vordergrund für die Vergabe des Preises. Er ist der älteste durch die Österreichische Gesellschaft für Dermatologie und Venerologie verliehene Preis", so die Präsidentin des ÖGDV Prof. Dr. Angelika Stary.

Scheck und Urkunden wurden gemeinsam von Vertretern Unilevers und der ÖGDV am 25. November 2016 im Rahmen der Jahrestagung im Marmorsaal des Hotel Sacher übergeben. „Wir freuen uns sehr über die herausragenden Arbeiten von Ass.-Prof. Dr. Patrick M. Brunner und Ph.D. Beate M. Lichtenberger. Beide sind würdige Preisträger ganz im Sinne Dr. Moriz Kaposis, der 1890 die Österreichische Gesellschaft für Dermatologie und Venerologie gründete. Wir gratulieren den beiden Wissenschaftlern. Außerdem möchte ich mich im Namen der ÖGDV bei Unilever für die so wichtige Unterstützung junger Dermatologie-Grundlagenforscher bedanken", so Prof. Angelika Stary.

Die entsprechende Förderung von Forschung und Entwicklung ist für das Unternehmen selbstverständlich.,Deshalb freue ich mich ganz besonders, diese wegweisenden Arbeiten der beiden Preisträger und ihrer Arbeitsgruppen mit unserer Auszeichnung zu honorieren. Im Namen von Unilever gratuliere ich herzlich", erklärte Ing. Gerhard Gribl von Unilever Austria, Manager wissenschaftliche Beratung/Bereich $R$ \& $D$.

\section{Atopische Dermatitis}

Ass.-Prof. Dr. Patrick M. Brunner, derzeit am Laboratory for Investigative Dermatology der Rockefeller University in New York tätig, fand heraus, dass man mit milden topischen Steroiden anti-entzündliche Effekte bei der atopischen Dermatitis erzielen kann. Mit vier- und 16-wöchiger Langzeit-Exposition mit dem Steroid Triamcinolone konnte in Neurodermitis-Zelllinien eine immunologische und genexpressive Reaktion erreicht werden. Das gibt die Hoffnung, diese Steroide eines Tages auch in einer LangzeitBehandlung der atopischen Dermatitis einzusetzen.

\section{Zelladhäsionsforschung}

Beate M. Lichtenberger, PhD, vom SERD (Skin \& Endothelium Research Division/Department of Dermatology/Medical University Vienna) erhielt den Preis für ihre Arbeit in der Zelladhäsionsforschung. Sie konnte nachweisen, dass epidermal Hedgehog und der Wachstumsfaktor TGF-b Veränderungen in der Dermis durch das Adhäsionsprotein Catenin bewirkt. Beta-Catenin induziert wiederum unter anderem atopische Haarfollikel. Mit pharmakologischen Methoden oder genetischem Knockout kann dieser von Catenin angeworfene Mechanismus gehemmt werden. Dies hängt jedoch von unterschiedlichen Parakrin-Antworten verschiedener Fibroblasten-Zelllinien ab, wie Lichtenberger erklärt. Hier tun sich also neue Wege in der Grundlagenforschung der Signalwege in Hautzellen auf.

hautnah $2017 \cdot 16: 14$

DOI 10.1007/s12326-017-0227-7

Online publiziert: 24. Januar 2017 C Springer-Verlag Wien 2017 